\title{
IV.
}

\section{Ueber Untersuchung von 73 Tanbstummen mit Rücksicht auf die Erscheinungen der "Otopiesis".}

(Vortrag, gehalten auf der 58. Versammlung deutseher Naturforscher und Aerzte in Strassburg.)

\author{
Yon \\ Dr. Roller \\ in Trier.
}

Meine Herren!

Wie Ihnen allen bekannt ist, hat, wie schon frther Herr Prof. Voltolini, auf dem dritten internationalen otologischen Congresse Herr Prof. Boucheron den sogenannten Ohrdruck, die Otopiésis, als Hauptmoment bei der Entstehung der Taubstummbeit präcisirt. In den allermeisten Fällen werde eine fast immer erst nach der Geburt erworbene Nasenrachenaffection zum Tubenabschluss fuhren. Die im Mittelohr eingeschlossene Luft werde langsam von den Blutgefässen absorbirt und infolge dessen entstehe ein Ueberdruck von aussen auf das Trommelfell, wodurch die Reihe der Gehörknöchelchen einen vermehrten Druck der Labyrinthflüssigkeit auszuïben gezwungen sei. Infolge dieses Druckes komme es zu Drucklähmung und Atrophie der Nervenfasern, wodurch die nicht wieder herstellbare Taubheit vollendet sei.

Der verderbliche Nasenrachenkatarrh nehme seinen Anfang: im Verlaufe von Scharlach, Masern, Diphtherie, Syphilis, Typhus oder auch einer gewöhnlichen Erkältung.

Die Anfangssymptome des Obrdruckes treten bald mit Fieber, bald ohne Fieber anf, bald unter Gehirnerscheinungen, bald ohne diese.

Von den objectiven Befunden am Ohre hebt Boucheron ganz ausdruicklich hervor, dass am äusseren Ohre, am Trommelfell und an den Gehörknöchelchen meistens nur normale Ver- 
hältnisse zu constatiren seien, ausser dass das Trommelfell häufig eingezogen sei und die Gegend des kurzen Hammerfortsatzes Injection der Gefässe zeige. Im Uebrigen constatirt er, dass Trommelfellperforation in keinem Zusammenhang mit der Taubheit stehe, sondern nur ein begleitendes oder nachfolgendes Symptombilde.

Die Nase, der Nasenrachenraum und die Eustachi'sche Trompete zeigten Sehwellungs- oder überhaupt Katarrhzustände. Der zu Anfang bestandene Schluss der Trompete könne sich zwar im Laufe der Zeit bessern, aber hebe sich selten völlig.

Die Hörprifung nimmt Boucheron recht genau auf die verschiedenste Art vor, mit allen möglichen Musikinstrumenten und constatirt dann auch, dass die Grade der Taubheit verschieden sind, dass in einzelnen Fällen noch Töne, in anderen keine Töne mehr gehört werden. Diesen verschiedenen Grad der Taubheit erklärt er aus dem verschiedenen Grad des Druckes, der auf die Gehörnerven einwirke.

Die Prognose richte sich nach der Dauer des Ohrdruckes. In frischen Fällen sei eine Wiederherstellung möglich und ihm factisch geglückt.

Seine Ansicht sucht Herr Boucheron zu stützen theils durch klinische Beobachtungen, theils durch anatomisch-physiologische Experimente, die er mit Erfolg an Hunden machte.

Obschon nun die Experimente der Herren Politzer und Bezold diesen Versuchen direct widersprechen, indem die Aquäducte schnell jede Druckschwankung der Endo- und Perilymphe ausgleichen und obschon es von vornherein unwahrscheinlich klingt, das vielgestaltete Bild der Taubstummbeit aus einem einzigen Punkte zu erklären, so verdient die Hypothese der Otopiésis dennoch ganz entschiedene Beachtung. Wenn sie sich als richtig herausstellte, wären die praktischen Erfolge ganz bedeutende. Wie jetzt schon fast jede Blindheit der Sänglinge, entstanden aus Conjunctivalblennorrhoe, mit Recht auf das Gewissen der Hebamme und des Arztes zurïckfällt und dieselben dem Richter verantwortlich macht, so müssten in Zukunft die meisten Fälle der Taubstummheit vermieden werden können, indem die Nasen-Rachenkatarrhe bei Typhus, Masern, Scharlach, Diphtherie und Syphilis der gewissenhaftesten Behandlung unterzogen werden miissten. Es würde daraus aufs Neue die Nothwendigkeit folgern, dass der praktische Arzt sich mehr mit der Ohrenheilkunde beschäftige, oder dass er wenigstens die Wich- 
tigkeit der Disciplin einsehen lerne und im gegebenen Fall, wenn seine Kenntnisse nicht ausreichen, einen sachverständigen Collegen zu Rathe ziehe.

Um mir in der Frage der Otopiesis zur Taubstummleit ein Urtheil bilden zu können, untersuchte ich das mir erreichbare Material nach dieser Richtung hin und erlanbe mir nun, Ihnen, meine Herren, die Resultate dieser Untersuchungen vorzulegen.

Bei der Untersuchung eines jeden einzelnen Taubstammen hielt ich folgenden Gang ein. Zuerst stellte ich anamnestisch fest die hereditären Verhältnisse, dann Dauer, Ursache und Verlauf der Erkrankung, ob Ohrenfluss, Schmerz, Schwindel, subjective Gehörgeräusche, frihere Gehörleiden vorhanden waren, ob sich in Bezng auf die Taubheit irgend etwas geändert hat. Dann prifte ich die Function der Ohren mittelst Uhr, Politzerschen Hörmessers, accentuirter Flüstersprache, Umgangssprache, Stimmgabel von verschiedener Höhe, Galtonpfeife, verschiedenartiger Geräusche, Höhrrohrs und besonders auf die verschiedenen Arten von Tönen mittelst einer grösseren Harmonika.

Gestatten Sie mir, meine Herren, einige Worte uber dieses Instrument als Mittel zur Prüfung der Tonempfindung beizufügen. Nachdem ich verschiedenfach bei Orgel- und Instrumentenbauern und Musikverständigen mich ansgesprochen hatte ther den Wunsch, ein Instrument zu besitzen, welches mich in den Stand setze, die Gehörfunction eines Menschen auf Töne der verschiedensten Höhe und Klangfarbe prïfen zu können, gab mir zuletzt einer der Herren den Rath, eine Harmonika zu diesem Zweck zu benutzen. Und, meine Herren, ich muss gestehen, dieses Instrument hat seine Vorzige. Ich bin damit im Stande, jeden Ton so lange zu halten, als ich will; ich kann ihn so stark und so schwach bervorbringen, wie ich will; mir stehen die hohen Töne und die tiefen Töne zu Gebote. Zudem ist die Kंlangfarbe der Töne eine ungleiche; die einen sind weich, zart, die anderen sind mehr metallisch. Dann besitzt das Instrument einen Vorzug, den die anderen Instrumente ausser der Orgel nicht haben, den ich aber für recht praktisch halte, nämlich dass ich durch Oeffnen einer besonderen Klappe im Stande bin, ohne Tonerzeugung das Instrument zu bewegen, als wenn ich Töne producire. So gelang es mir öfters, Selbsttäuschung zu entdecken. Jedenfalls hat das Instrument den Vorzug, compendiös zu sein. Auch lässt es sich zu vergleichend wissenschaftlichen Prifungen sehr wohl verwen- 
den, wenn man nur die einzelnen Töne von einem Musiker bestimmen lässt.

Die objective Untersuchung erstreckte sich auf die Ohrmuschel, den äusseren Gehörgang, die Durchgängigkeit der Ohrtrompete, das Trommelfell, dann auf die Nase, den Nasenrachenraum und auf die allgemeine Körperconstitution.

Die Diagnose auf Labyrinthaffection setzte ich fest nach ausgiebiger Prufung der Tonperception durch Luft- und Knochenleitung. Ich begnügte mich nicht mit der Priffung versehiedener Stimmgabeln, weil ich fand, dass die meisten meiner untersuchten Taubstummen diese sebr wohl pereipirten, dagegen bei anderer Pruffung bedeutende Defecte zeigten.

Ich untersuchte im Ganzen 73 Taubstumme, 42 männliche und 31 weibliche. Das Alter sehwankte vom 6. bis 69. Jahre. Die meisten waren 12,13 und 14 Jahre alt.

Von diesen 73 Taubstummen waren bei nur dreien beiderseits keine Labyrinthaffectionen vorhanden, $d$.h. sie vermochten alle Töne, von den höchsten bis zu den tiefsten, zu percipiren, entweder in der Luftleitung aus der Nähe des Ohres und mittelst eines Höhrrohres oder nur durch Knochenleitung, indem ich die Tonquelle entweder an das Schläfenbein oder an das Hörrohr anlegte.

Bei vier anderen war einseitig das Labyrinth frei. Bei allen Anderen lag eine Labyrinthaffection vor. Von diesen 66 Tanbstummen wurden 8 beiderseitig, von einem nur halbseitig jede Perceptionsprifung des Gehörnervenapparates negativ beantwortet. Es wurde weder Uhr, noch Politzer's Hörmesser, noch irgend welche Stimmgabel in der Knochenleitung vernommen. Ebensowenig wurde die Galtonpfeife oder die Harmonika percipirt, nicht einmal in den tiefsten Tönen und zwar im Knochencontact. Bei diesen Taubstummen ist also mit Sicherheit anzunehmen, dass der Nervenapparat in der Schnecke völlig ans seiner Function ausgeschaltet ist.

Bei den restirenden 57 Taubstummen ist beiderseitig und bei einem noch einseitig wenigstens eine beschränkte Thätigkeit des Schneckennervenapparates vorhanden. In der Luftleitung wurde die'Uhr von keinem dieser 58 gehört, Politzer's Hörmesser wurde in 6 Fällen auf beiden Seiten und in 2 Fällen einseitig gehört, und zwar entweder dieht am $\mathrm{Ohr}$ oder in einzelnen Fällen bis zu $20 \mathrm{Cm}$. Entfernung; accentuirtes Flustern wurde in keinem Fall gehört; die Umgangssprache oder, colrecter 
ausgedrüickt, Tonlaute mit der Stärke der Umgangssprache wurden in 4 Fällen auf beiden Seiten gehört. Diese Taubstummen, mit der nöthigen Vorsicht untersucht, hörten unzweifelhaft meine Worte, zwei selbst auf $30 \mathrm{Cm}$. Entfernung und sprachen sofort nach, aber nur bei dem Worte Papa oder höchstens Mama. Bei anderen Worten sprachen sie irgend ein Wort aus, was in den Vocalen eine entfernte Aehnlichkeit mit dem vorgesprochenen hatte. Diese Thatsache ist interessant. $\mathrm{Ob}$ nun die Kranken nicht geübt genug sind, um auch die Worte nachzusprechen, welche complicirter sind als Papa, oder ob sie nur einzelne Vocale und einzelne Consonanten hören, das ist schwer festzustellen, jedenfalls hörten sie wenigstens Laute.

Mit Hülfe des Hörrohres hörten nur 20 die eben erwähnten Wortlaute, die sie ohne Hörrohr überhaupt nicht gehört hatten.

In der Knochenleitung wurde Politzer's Hörmesser in 12 Fällen von den 58 Taubstummen gar nicht vernommen, in 5 Fällen einseitig und in 41 Fällen doppelseitig, aber selbstverständlich verschieden stark, so dass ich bei einzelnen im Unklaren war, ob sie nicht vielleicht nur eine Erschütterung vernommen hätten und nicht einen Ton. Die Uhr wurde in 5 Fällen, aber nur einseitig vernommen. Die betreffenden Kranken machten mit den Fingern die rasche Bewegung des Tickens der Uhr nach, so dass ich in keinem Zweifel über das Resultat war. Die Stimmgabel wurde von den 58 Kranken percipirt und zwar s0. wohl mit hohen, als auch mit tiefen Tönen, ebenfalls verschieden intensiv. Die Stimmgabel wurde vom Scheitel ans in 19 Fällen nicht im Ohr percipirt, sondern $18 \mathrm{mal}$ auf dem Scheitel selbst und 1 mal nur im Kehlkopf. Dass die Empfindung eine Tonempfindung war und nicht lediglich die Empfindung des Fibrirens, konnte ich deutlich an dem plötzlich auftretenden freudigen Erschrecken der Personen wahrnehmen. In 2 Fällen wurde sie überhaupt nicht percipirt, während dies am Proc. mast. wohl der Fall war. In den übrigen 37 Fällen wurde die Stimmgabel bald nach rechts, bald nach links hin besser vernommen.

Die Galtonpfeife wurde von keinem der untersuchten Taubstummen wahrgenommen.

Hieraus ersieht man, dass der Grad der Erkrankung im Nervenapparat des Ohres ein verschiedener ist. Darin stimmen jedoch alle Fälle überein, dass die hohen Töne nicht, wohl aber die tiefen Töne wahrgenommen werden. Nach dem heutigen Stand ${ }^{\circ}$ der Wissenschaft ist man berechtigt, anzunehmen, dass 
die erste Schneckenwindung die höheren Töne repräsentirt, dass demnach beim Ausfallen der höheren Töne in der ersten Schneckenwindung ein pathologischer Zustand vorliegen muss.

Welche Töne speciell ihr Perceptionsorgan daselbst besitzen, muss sich durch vergleichende Untersuchungen am Lebenden und bei der Obduction vielleicht feststellen lassen. Ich verfüge zufällig über eine Anzahl Taubstummer in unserer Anstalt und werde ich mir es zur Aufgabe machen, die Schwingungszahl der Töne festzustellen, welche noch vernommen werden, um später durch die Obduction orientirt zu werden uber die Localisation der Töne in der Schnecke.

Die angefthrte Thatsache steht in Uebereinstimmung mit den Angaben Boucheron's und anderer Autoren, so von den Herren Moos und Steinbrügge, welche ja nachwiesen, dass bei Taubstummen die Nerventheile der ersten Schneckenwindung Atrophie zeigten.

Neben Labyrintherkrankung fand ich in den meisten Fällen eine Miterkrankung des Mittelohres.

Eine Mittelohrerkrankung nahm ich an, wenn ich pathologische Zustände im Gewebe des Trommelfells und Adhäsivprocesse vorfand. Zur Feststellung der Diagnose war ich allein auf die Untersuchung mit dem Ohrspiegel und Siegle'schen Trichter und auf das Ergebniss der Luftdouche mit Bezug auf das Trommelfell angewiesen. Die Auscultation während der Luftdouche konnte ich nicht verwerthen, weil die Ergebnisse sich auf die Zustände der Tuba Eustachii bezogen und etwaige Geräusche im Mittelohr verdeckten. Die Gehörprüfungen konnte ich nicht verwerthen, weil sie bei der gleichzeitig vorhandenen Labyrinthaffection keinen Anhaltspunkt geben, in wie weit die Resultate auf das Labyrinth und in wie weit auf das Mittelohr zu beziehen sind.

Bei den 73 untersuchten Taubstummen fand ich das Trommelfell nur 13 mal beidseitig und zweimal einseitig normal, so dass also 58 Taubstumme beidseitig und zwei nur einseitig pathologische Zustände zeigten. Die Trommelfelle boten, selbstverständlich in verschiedenem Grad, verschiedene Befunde, wie sie sich nach abgelaufenen oder bei noch subacut bestehenden Mittelohrkatarrhen oder Entzündungen vorfinden. Matt, wie bestaubt, häufig mit abnormem Glanze, häufiger ohne Glanz, von grauer Farbe mit wechselnder Niiancirung ins Gelbe oder schmutzig Rothe, mit hellglänzenden sehnigen Streifen und Kalk- 
concretionen besetzt, theils mit dicken, theils dunnen Narben häufig versehen. Die Durchsichtigkeit war nur an einzelnen Partien vorhanden. Der Hammer in allen Fällen sichtbar, aber in allen Fällen auch von der Norm abweichend. Der Proc. brev. stark vorspringend, entweder glänzend weiss oder häufiger verbreitert und alte chronische Injection der Blutgefässe zeigend. Seine Ligamente scharf vorspringend. Fast nie vermisste ich den sehnig glänzenden, undurchsichtigen, halbmondförmigen Streifen, der vom Proc. brev, sich am oberen hinteren Rande des Trommelfells hinzieht und welcher auch in der letzten Zeit von Bezold bei seiner Untersuchung der kindlichen Gehörorgane hervorgehoben wurde. Der Hammergriff war meist verbreitert, injicirt und mit breitem, undurehsichtigem Saum umgeben. Seine Spitze stets verbreitert. In circa vier Fällen fand ich das Trommelfell mit dem Promontorium der Paukenwand verwachsen. In fünf Fällen war das Trommelfell versehwunden oder perforirt. Die vorliegende Paukenschleimhaut war in einem Falle sehnig glänzend, in anderen Fällen gewulstet, geröthet und mit Secret bedeckt.

Die Einziehung des Trommelfells in pathologischem Maassstab, mitunter so, dass der Hammergriff horizontal stand, zeigten 66 Untersuchte beiderseits, einseitig noch zwei.

Der Reflex war in einlzenen Fällen normal, in den meisten Fällen pathologisch und zwar entweder abnorm glänzend oder ganz matt, an nicht gewöhnlichen Partien des Trommelfells auftretend, so am Rande oder an den Ligamenten des Proc. brev. oder in mehrfachen Parallelstrichen sich zeigend.

In 27 Fällen zeigte der Siegle'sche Trichter eine normale Beweglichkeit des Trommelfells in allen seinen Theilen. In allen anderen Fällen war die Bewegung des Trommelfells entweder eine sehr träge, schwerfällige oder es bewegten sich nur einzelne Partien, während der Hammer unverändert blieb, oder es trat ein Flattern. der dünnen Trommelfellnarben auf.

Die Luftdouche verursachte nur in einem Falle ein Vorspringen des Trommelfells und in einem Falle eine Injection des Hammergriffs. In allen anderen 71 Fällen war die Reaction gleich Null.

In vier Fällen war permanente Ohreiterung vorhanden.

Nur in vier Fällen konnte ich bei ganz normalem Trommelfell, völliger Durchsichtbarkeit, völliger Beweglichkeit bei Siegleschem Trichter and Luftdouche und bei Abwesenheit jeglichen 
Nebengeräusches jede Abwesenheit von Mittelohrprocessen annehmen; in den 69 anderen Fällen liegen unzweifelhaft schwere pathologische Processe vor. Dieselben sind hüchstwahrscheinlich in den Fällen mit erhaltenem Trommelfell die Ueberreste von friher abgelaufenen katarrhalischen oder entzuindlichen Processen, bestehend in Ankylosen der Gehörknöchelchen, in narbigen Verwachsungen derselben unter einander und mit der Paukenwand und in narbigen Verwachsungen der Stapesplatte und narbigem Verschluss der Fenestra rotunda. Einen Fall letzterer Art bekam ich zur Obduction und erlaube ich mir denselben hier kurz za erwähnen.

Ursula Kaltenberg, 40 Jahre alt, vom Lande, war von Kindheit an auf beiden Seiten taub und beinahe stumm. Die 'Taubheit trat nach einer acuten Erkrankung auf unter starken Kopfschmerzen. Ohrenfluss war nie vorhanden; Schmerz dagegen, Schwindel und starke subjective Gehörsempfindungen traten häufig auf. Erbliche Belastung konnte ausgeschlossen werden. Die Prufung der Gehörfunction ergab links in der Luftlinie Perception des Politzer'sehen Hörmessers auf nur $5 \mathrm{Cm}$., rechts die Perception von Politzer's Hörmesser und der Uhr am Ohre. Die Luftdouche übte keinen verbessernden Einfluss ans weder auf die Function, noch auf die Ohrgerătusche.

In der Knochenleitung wurde Politzer's Hörmesser gut, ebenso die Stimmgabel vernommen, wenn auch die hohen Töne schlechter. Die Uhr wurde schwach am Proc. mast., gar nicht auf dem Scheitel vernommen. Der R in ne'sche Versuch gelang positiv; Galton's Pfeife wurde gar nicht gehört. Tiefe Töne wurden in der Luftleitung wahrgenommen, hohe nicht. An der äusseren Ohrmuschel, am Meat. aud. extern. und am Trommelfell wurde kein pathologischer Zustand festgestellt. Bei Anwendung des Siegle'schen Trichters bewegten sich nur einzelne Partien am Trommelfell, nicht aber der Hammer, welcher unbeweglich blieb. Bei Luftdouche hörte man beiderseits ein dünnes Giemen mit trockenen Rasselgeräuschen. Dabei bewegte sich das Trommelfell nicht, aber es trat eine lebhafte Röthe an dem. selben besonders am Hammergriff auf. Patientin litt gleichzeitig an Ozaena und Catarrh des ganzen Rachengewölbes, welches dicht mit Borken bedeckt war.

Patientin starb an einer intercurrenten Krankheit. Die Obduction ergab gleichmässig in beiden Pankenhöhlen dichte, feste Verwachsung des Stapes in der Fenestra oval., so dass er ganz in Narbengewebe eingebettet war. Ebenso war die Fen. rotund. völlig ausgefüllt mit Narbengewebe. Im Uebrigen war in der ganzen Paukenhöhle nichts Abnormes nachzuweisen. Die Tub. Eust. war auf beiden Seiten verengert. Am Labyrinth war makroskopisch ein pathologischer Zustand nicht nachzuweisen. Ich reservirte die Theile zur genauen mikroskopischen Untersuchung. 
Ein gleicher anatomischer Zustand wird sich unzweifelhaft bei manchen Taubstummen finden. Jedenfalls werden fibröse Fäden oder Membranen, wie ich sie häufig genug bei der Obduction von Sehwerhörigen fand, in den meisten Fällen die Ursache der abnorm tiefen Einziehung und geringen Beweglichkeit des Trommelfells sein.

Um kurz zu recapituliren: von 73 untersuchten Taubstummen hatten 70 eine Labyrintherkrankung, drei waren frei davon und hatten nur eine Mittelohrerkrankung und nur vier waren frei von derselben und hatten nur eine Labyrintherkrankung.

In diesem Resultate, in der Seltenheit von Taubheit ohne Erkrankung der Paukenhöhle, befinde ich mich in Uebereinstimmung mit anderen Forschern, aber im Widersprueh mit Boucheron, weleher hervorhebt, dass am äusseren Ohr, am Trommelfell und an den Gehörknöchelchen meistens nur normale Verhältnisse zu constatiren seien, dass dagegen das Trommelfell fast immer stark eingezogen sei und nur eine Injection des Proc. brev. zeige. Trommelfellperforationen etc. seien nur ein begleitendes oder nachfolgendes Symptom.

Ich gehe nun weiter und behaupte, dass die in den allermeisten Fällen constatirte Labyrinthaffection nicht Folge des Ohrdruckes, der Otopiesis, ist, sondern Folge der Mittelohrerkrankung.

Wie Boucheron, constatirte auch ich in 63 Fällen von 73 Untersuchten einen pathologischen Zustand der Tub. Eustach., deren Passage mehr oder weniger verlegt oder selbst ganz geschlossen war (in zwei Fällen). Das Durchpressen von Luft erforderte eine ziemliche Kraft und nie vernahm ich das leichte Anschlagegeränsch, sondern ein dünnes Pfeifen oder Giemen, vermischt mit trockenem and feuchtem Rasseln.

So unzweifelhaft die Eröffnung der Tubenpassage auf die Verbesserung der Gehörfunction wirkt, so selten sind doch die bekannt gewordenen Fälle von geheilter Taubheit durch Aufhebung des Tubenverschlusses, so selten, dass es mir natürlicher erscheint, die Fälle von wirklicher Taubstummheit, bei denen eine Labyrinthaffection vorliegt, dadurch zu erklären, dass das Mittelohr durch Erkrankung seiner Gewebe- und Organelemente die weitere Erkrankung des Labyrinths veranlasste und nicht ein abnormer Druck der Gehörknöchelchenreihe auf die Labyrinthfliussigkeit. Wie schon frtiher erwähnt ist, widerspricht die anatomische Thatsache der Annahme, als wenn ein einseitiger Druck auf die Labyrinthflïssigkeit im Stande wäre, einen mehr 
als physiologischen Reizdruck auszuüben, indem die Aquäducte eine fortwährende Erneuerung der Endo- und Perilymphe besorgen. Es widersprechen auch die Experimente von Politzer und Bezold dieser Annahme.

Anders liegt die Sache, wenn winannehmen, dass das Mittelohr zuerst erkrankt. Wie leicht erklärt die anatomisch oft constatirte Thatsache der Steigbuigelankylose und des narbigen Verschlusses der Fenestra rotunda die Taubheit und erklärt auch in plausibler Weise die anatomisch constatirte Thatsache der Atrophie der Nervenendigung in der ersten Schneckenwindung. Wenn ein entzündlicher Process an der Fen. rot. sich etablirt hat, dann wird nach jeder Analogie irgend eines Körperorgans der in dichtester Nähe gelegene Organtheil unzweifelhaft mit erkranken. Wenigstens wäre die Annahme, dass die Entzündung an der Paukenseite des zweiten Trommelfells seinen Abschluss fände, ohne irgendwie nach der anderen Seite überzugreifen, anatomisch unlogisch. Auch lehrt die Untersuchung von Burkhardt-Merian, dass eine Belastung des runden Fensters die hohen Töne verschwinden lässt. Ein Verschluss des runden Fensters mit einem Bindegewebspfropf dürfte wohl denselben Effect herbeiführen. Dass ferner ein Nervenapparat, wie der im Labyrinth, wenn bei Verschluss des runden und ovalen Fensters keinerlei Erregung mehr einwirken kann, langsam der Atrophie verfällt, ist eine uns ganz geläufige Thatsache und wird auch durch meine Untersuchungen unterstützt, indem die von mir untersuchten älteren Taubstummen von über 40 bis 60 Jahren gar keine Tonperception zeigten, ausser denjenigen, welche noch nicht absolut taub waren, sondern noch grobe Geräusche vernahmen, so beim Zuschlagen einer Thür oder noch einzelne Töne einer Orgel. Bei einem dieser Taubstummen fand ich dann noch, um die ausgesprochene Ansicht annehmbarer zu machen, bei einer forcirten Anwendung des Siegle'schen Trichters, dass die Patientin wie vom Blitz getroffen niedersank, nach Wiederkehr des Bewusstseins auf 24 Stunden noch über Schwindel klagte und erbrach. Ich glaube keinen Fehlschluss zu thun, wenn ich annehme, in diesem Falle war das Tymp. secund. oder die Steigbtigelplatte noch einer gewissen Bewegung fähig, ,wodurch die Schnecke nicht der völligen Atrophie verfallen konnte.

Auch die Aetiologie unterstutzt die Annahme, dass destruirende Processe im Mittelobr die ursprüngliche Ursache der Taubheit sind. Von allen Obrkrankheiten, die zur Schwerhörigkeit 
führen, sind die der Paukenhöhle die am häufigsten vorkommenden.

Von meinen Untersuchten wird die Erkrankung hergeleitet in 5 Fällen von Scharlach, in einem Fall von Scharlach mit Diphtherie, in 14 Fällen von einer Erkrankung, die mit Krämpfen und meningitischen Erscheinungen verlief, in 11 Fällen von Typhus, in 3 Fällen von Masern und Rötheln, in einem Fall von einer Geschïtzdetonation, in einem Fall von Schnaps, nach dessen Genuss ein sechsjähriges Kind betrunken hinfiel und nach dem Erwachen schwerhörig war und dann völlig taub wurde, in 2 Fällen von einer acuten Ohreiterung und in 35 Fällen liess sich der Grund nicht mehr feststellen. In 24 Fällen wurde die Erkrankung von Geburt an constatirt, d. h. in der Säuglingszeit, damit ist aber nicht ausgeschlossen, dass nicht ein Krankheitsprocess in den ersten Wochen dennoch die Ursache der Taubheit bildet. In 47 Fällen trat die Taubheit in den späteren Jahren ein.

Nun ist es ja bekannt, wie häufig Mittelohrerkrankungen im Verlaufe von Masern, Scharlach, Rötheln, Diphtherie und Typhus vorkommen, so dass man nicht zum Tubenverschluss greifen muss, um eine Erklärung für entstehende Taubheit zu finden.

Die 4 Fälle von Taubheit, die nur auf einer Erkrankung des Labyrinths basirten und nicht mit einer Affection des Mittelohres verbunden waren, rührten in 2 Fällen her von einem überstandenen Typhus, in einem Fall von einer Meningitis und in einem Fall war die Taubheit angeboren. In diesen Fällen lag die Ursache entweder in einer centralen Erkrankung oder in einer localen Affection des Labyrinths. Also auch hier ist die Erklärung dureh Otopiesis überflussig oder bietet keinen Anhaltepunkt, weil gerade das Trommelfell ganz flach und die Tube völlig passirbar war.

In Bezug auf erbliche Belastung konnte ich erfahren, dass in 2 Fällen Blutsverwandtschaft zwischen den Eltern bestand und dass in 9 Fällen andere Geschwister oder nahe Verwandte ebenfalls taubstumm waren. In diesen 9 Fällen war aber nachzuweisen, dass jedesmal die Krankheit acquirirt wurde im Verlaufe einer der oben genannten Krankheiten, so dass mehrmaliges Vorkommen von Taubheit in der Familie reiner Zufall ist oder vielmehr nur darauf beruht, dass der scrophulöse Habitus oder die Disposition zu Rachen- und Mittelohrerkrankungen in der Familie lag. 
Für die Annahme, dass Mittelobrerkrankungen die Ursache der Taubheit in meinen Fällen in den allermeisten Fällen bieten, spricht die Thatsache, dass diese Taubstummen auffallend häufig in meine Behandlung gelangten wegen intereurrenter Mittelohrerkrankung, wegen perforativer Entzïndung, Katarrh, Nekrose und Cholesteatom des Antr. mast., welehes in einem Fall zur operativen Eröffnung des Proc. mast. fuhrte.

Ferner spricht fitr die Richtigkeit meiner Ansicht die Thatsache, dass die taubstummen Kinder und selbst die Erwachsenen auffallend häufig Serophulose zeigten, aufgeworfene Lippen, dicke Nase, Ekzeme und Nasenrachenkatarrhe, also disponirt waren zu. Mittelohrerkrankung und zwar gerade zu den schlimmeren Formen derselben.

Dass der Weg dazu durch die Tuba Eust. von der Nase und dem Rachen ausgehen kann und in vielen, vielleicht den meisten Fällen ausgehen wird, ist bei Scrophulösen sehir klar. In 38 Fällen von meinen Untersuchten fand ich ehronische Schwellungszustände der Nase und des Rachens.

Aus meinen Untersuchungen ziehe ich folgende Schlüsse:

1. Nicht ein Fall der von mir untersuchten deutet auf die Erscheinungen der Otopiesis.

2. In der grössten Mehrzahl der Untersuchten lässt sich die Taubstummbeit nach dem vorhandenen objectiven Befund ungezwungen aus abgelaufenen Mittelohrprocessen erklären.

3. Die vorgefundene Labyrinthaffection entwickelte sich erst secundär aus den Mittelobrprocessen.

Praktisch wichtig erscheint das Resultat deshalb, weil eine Anzahl Ohrkranker bei sachgemässer Behandlung vor völliger Taubheit bewahrt bleiben können. Wenn dies Wahrheit ist, tritt an den praktischen Arzt die unabweisbare Pflicht heran, davon Kenntniss zu nebmen. 